\title{
Low coverage palladium adsorption on graphene: first principles study
}

\begin{abstract}
In this paper, we investigate stable geometries, electronic and magnetic properties of low coverage palladium (Pd) atom adsorption on graphene using first principles calculations with the generalized gradient approximation. Calculations show that single Pd atom located at the top of carbon atom is the energetically favorable configuration, and is found to be semiconductor and non-magnetic. We also compute the projected density of states (PDOS) around Fermi level and beyond. It is found that, $\mathrm{C}$-Pd covalent interaction is mainly dominated by $2 \mathrm{pz}$ of $\mathrm{C}, 5 \mathrm{~s}$ and $4 \mathrm{~d}$ like states of $\mathrm{Pd}$. For low coverage stable Pd dimer, the adsorption is characterized by strong hybridization between the palladium atoms and the two carbon atoms bonded directly to it. A much weakening of $\mathrm{Pd}-\mathrm{Pd}$ bond is observed and the C-Pd covalent bonds mainly dominate by $2 \mathrm{pz}$ of $\mathrm{C}$ orbital indicating that planar coating can be achieved. Thus, this work reveals that uniform coating of $\mathrm{Pd}$ atom can be achieved and may be useful in transport measurements.
\end{abstract}

Keyword: Density functional theory; Electric; Geometric; Graphene; Magnetic properties 